\title{
Retroreflector array as a tapped delay-line filter for ultra-short optical pulses
}

\section{A. Sabatyan \\ sabatyan@iasbs.ac.ir}

J. Jahns
Institute for Advanced Studies in Basic Sciences(IASBS), P.O.Box 45195-1159, Zanjan, Iran

FernUniversität Hagen, Optische Nachrichtechnik, Universitätsstr. 27/PRG, 58084 Hagen, Germany

A retroreflector array can be used to act as an optical tapped delay-line filter for temporal optical signals. Here, we demonstrate the basic operation and present a theoretical description. Our theory is based on the mathematical formalism for finite impulse response filters. A relationship to the classical Talbot bands experiment is established. By adding a mask to the retroreflector array, one can implement arbitrary filter operations. As a specific example, a differentiation in frequency domain is demonstrated by using a simple binary mask. The proposed devise maybe useful for the optical filtering and shaping of ultra short pulses. [D0I: 10.2971/je0s.2006.06022]

Keywords: 0ptical communications, signal processing, all optical devices, microstructure devices, pulse shaping, micro-optics devices

\section{Introduction}

Ultra-short optical pulses with pulse widths in the fs-range are becoming increasingly important for a number of applications. An example is optical communications, both for long as well as short distances. For long-distance transmission fspulses may be used in time division and/or wavelength division multiplexing systems to enhance the transmission capacity. Recently, the "frequency comb" has opened up new possibilities for such transmission systems [1]. For short distance communications, e.g. optical interconnections for computers, the use of fs-pulses may help to optimize the performance of a data link [2].

Consequently, there is a need for components or modules that allow one the filtering of optical signals consisting of fspulses. Different types of devices may be used for this. One of the most powerful devices is the arrayed waveguide grating (AWG) [3]. Also under investigation are ring resonators [4], double grating devices [5], etc. In terms of linear systems theory, these may be categorized into finite impulse response filters (AWG and double grating setup belong into this class) and infinite impulse response filters (which includes ring resonators and Fabry-Perot type devices).

The hardware implementation of filter devices has to satisfy certain requirements: compact size, ruggedness, and tunability, are some of the main features. The physical dimensions that are required for a filter depend on the time delays that have to be generated. For a filter operating in the domain of the optical spectrum, this requires path length differences of only a few wavelengths. Recently, lithographic fabrication and the MEMS technology have allowed one to fabricate unusual optical elements. One can make devices that implement very small path length difference on the order of $1 \mu \mathrm{m}$ as in the case of a simple phase grating. Or one can use deep etching or micromachining to make deep structures of hundreds of micrometers, namely a retroreflector array (RA). The RA was fabricated in aluminum by ultraprecision micromachining. It consists of a periodic array of facets that form a right angle with each other. In the next section we will show how such an element can be used as an optical tapped delay-line.

\section{P TICAL SET UP}

The optical setup that we use, is shown in Figure 1.

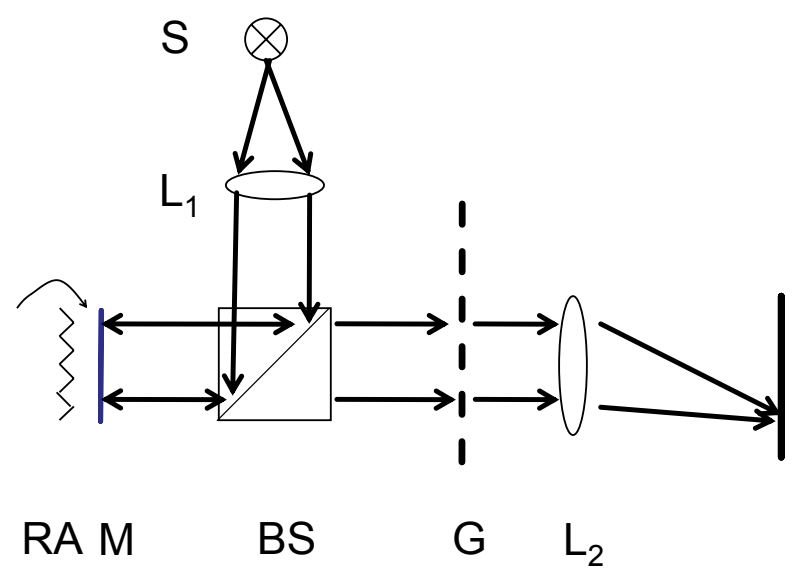

FIG. 1 Setup for generating the tapped delay-line with a retroreflector array RA. Its effect on the optical spectrum is observed by means of a grating $G$. $S$ is a light source, BS a beam splitter used to illuminate the RA, $L_{1}$ and $L_{2}$ are lenses. $M$ is a mask to implement specific filter function.

An RA with facets of equal sizes $\mathrm{W}$ is illuminated by a collimated beam. Facets are assumed to be oriented orthogonally 
to the plane of the drawing. Due to the $90^{\circ}$ angle between its sidewalls, each facet reflects a beamlet back into the direction of the incoming beam. However, if the orientation of the RA is normal to the optical axis, the path lengths for the different beamlets are identical. This is shown in the more detail in Figure 2-(left) which shows the situation for normal incidence $\left(\alpha=0^{\circ}\right)$ for a single facet of the RA.
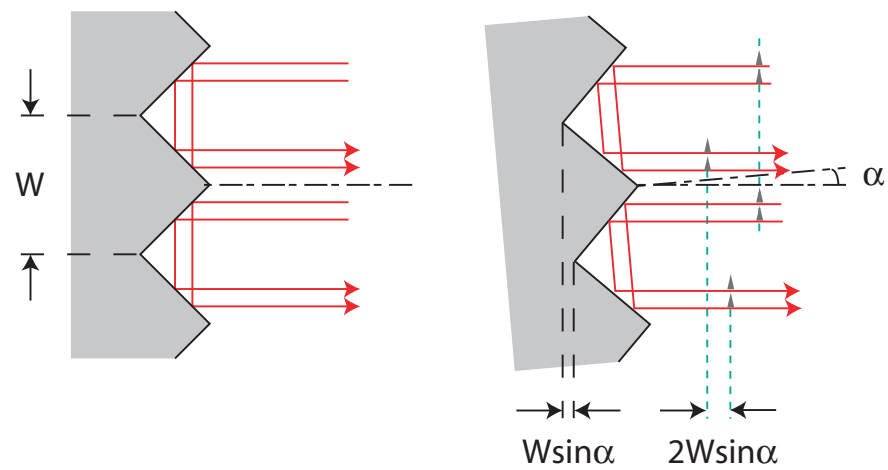

FIG. 2 Generating of time delays by means of the retroreflector: for normal incident (left), all the paths are identical. For a tilt angle $\alpha$, a different path lengths occur between different facets. Corresponding points in two neighboring are separated by a distance $W \sin \alpha$ in the direction of the optical axis. (W is the pitch of the RA.) Therefore, upon a reflection a total path length difference of $2 W \sin \alpha$ occurs.

If the RA is rotated around the axis, the optical path lengths will vary from one facet to the next. In Figure $2 b$ the situation is depicted for the case $\alpha>0$. Hence a temporal delay is introduced between the beamlets reflected from the different facets. The path length difference for two incoming beams that hit corresponding points of the $\mathrm{K}$-th and $(\mathrm{K}+1)$-th facet is $W \sin \alpha$. The same path length difference occurs for the outgoing beams. Hence the total time delay between neighboring facets is given as

$$
\tau_{r}=2 W \sin \alpha / c,
$$

where $c$ is the speed of light. Note that for two beams reflected from the same facet no time delay occurs. As an example, we used $W=640 \mu \mathrm{m}$ and $\alpha=1^{\circ}$. With these values we obtain $\tau_{r} \approx 74.5$ fs. In the extreme case we might tilt the RA by $\alpha=45^{\circ}$, so that one of the two sidewalls of each facet is normal to the incident beam. In this case, the generated time delay becomes maximal, namely $\tau_{r}\left(45^{\circ}\right)=\sqrt{(2) W / c}$. Using the same value for $W$ as above, we obtain a delay of 3 ps. The total number $\mathrm{K}$ of facets may typically range between 10 and 100 depending on the aperture of the RA and the optical setup. The maximum time delay generated by the two extreme facets of the RA is $\tau_{\text {total }}=K \tau_{r}$. The numerical examples show that by using suitable parameters one can turn the time delays over a range of two orders of magnitude. For small tilt angles the delays lie in fs-regime so that the optical spectrum is affected and we can visualize the effect by using a grating setup (see Figure 1). In this article we want to report about several such experiments. Before that, however, we will develop a suitable theory that also allows one to calculate the intensity in the optical spectrum.

\section{RETROREFLECTOR AS A TAP PED DELAY-LIN E : DESCR I P T I O N THE O R E I C A L}

In previous section we used a basic physical explanation for the occurrence of time delays generated by the tilted RA. From that starting point, it is easy to understand that the RA represents a tapped delay-line (TDL), also known as finite impulse response filter. Its general structure is shown in Figure 3.

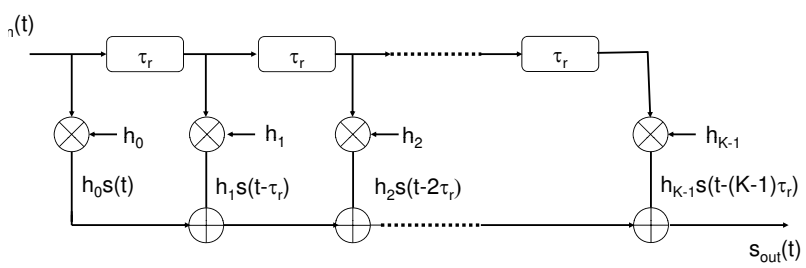

FIG. 3 Structure of a tapped delay-line filter. The input signal $s_{i n}(t)$ in divided into $\mathrm{K}$ branches, each with a time delay $\tau_{k}, k=0 \ldots K-1$, note that $\tau_{0}=0$.

A TDL splits an incoming signal $s_{i n}(t)$ into $K$ branches with a delay $\tau_{K}$ in the $K$-th branch. Here, we assume that all the time delays are identical, i.e.

$$
\tau_{k}=\tau_{r}
$$

In addition to the time delays a TDL applies different weights $h_{k}(k=0 \ldots K-1)$ to the different branches. The output signal $s_{\text {out }}(t)$ is then formed by summing up over all branches. Hence, the TDL is mathematically described by a discrete convolution [6]

$$
\begin{aligned}
s_{\text {out }}(t) & =\sum_{k=0}^{K-1} h_{k} s_{\text {in }}\left(t-k \tau_{r}\right) \\
& =h(t) * s_{\text {in }}(t),
\end{aligned}
$$

where $\mathrm{t}$ is the time coordinate and " $*$ " denotes the convolution operation. $h(t)$ is the temporal impulse response of the filter

$$
h(t)=\sum_{k=0}^{K-1} h_{k} \delta\left(t-k \tau_{r}\right),
$$

$K$ denotes the number of the facets of the RA. So far, we have explained how the time delays are implemented. However, it is also possible to implement different weights as required to implement a tapped delay-line. This can be achieved by positioning a mask in the plane of the RA. The mask may be a simple amplitude mask, we can implement real-valued, nonnegative filter coefficients. By using a phase mask, we can implement complex-valued coefficients and, in particular, also real-valued negative coefficients. In general, we can write

$$
h_{k}=\left|h_{k}\right| \exp \left[i \arg \left(h_{k}\right)\right]
$$

As a specific example, we consider the case where coefficients are real and increase linearly with $k$ as it is shown in Figure 4. 


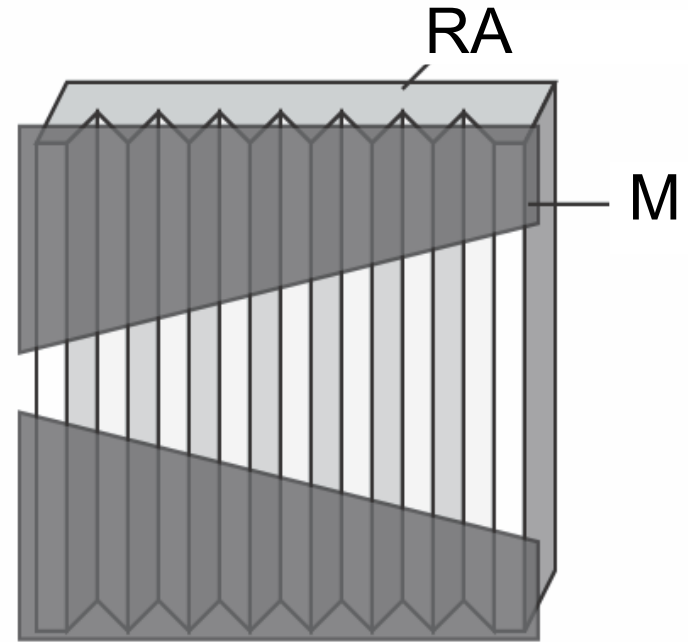

FIG. 4 By inserting a binary mask $\mathrm{M}$ of variable height, different weight factors $h_{k}$ can be implemented.

For this example, we can write

$$
h_{k}=h_{0}+k \Delta h
$$

In this case, the value of the $k$-th coefficient is represented physically by the height of the mask across the $k$-th facet.

In frequency domain, the transfer function $H(v)$ is related to the impulse response through a Fourier transformation

$$
H(v)=\int h(t) \exp (2 \pi i v t) \mathrm{d} t .
$$

So that the spectrum of the output signal, $S_{\text {out }}(v)$, is given as the product of the signal spectrum, $S_{i n}(v)$, and the transfer function

$$
S_{\text {out }}(v)=H(v) S_{\text {in }}(v)
$$

Since impulse response is discrete, Eq. (4), we obtain a periodic transfer function. We can write

$$
H(v)=\sum_{k=0}^{K-1} h_{k} \exp \left(-2 \pi i k v \tau_{r}\right)
$$

As we will show in the next section on experiment results, indeed we obtain a periodic modulation of the spectrum. And for our special example, Eq. (6), the following transfer function results

$$
\begin{aligned}
H(v) & =\sum_{k=0}^{K-1}\left(h_{0}+k \Delta h\right) \exp \left(-2 \pi i k v \tau_{r}\right) \\
& =h_{0} F(v)+\frac{i \Delta h}{2 \pi \tau_{r}} \frac{\partial F(v)}{\partial v}
\end{aligned}
$$

where $F(v)$ is defined as

$$
F(v)=\sum_{k=0}^{K-1} \exp \left(-2 \pi i k v \tau_{r}\right) .
$$

Hence with simple means we can implement, for example, a differentiation in the spectral domain. However, also other operation are possible.

\section{EXPERIM E N TAL RES ULTS}

In our experiment, an RA with a pitch $\mathrm{W}=640 \mu \mathrm{m}$ was used. The RA was fabricated by diamond cutting in aluminum. A photograph of it is shown in Figure 5.

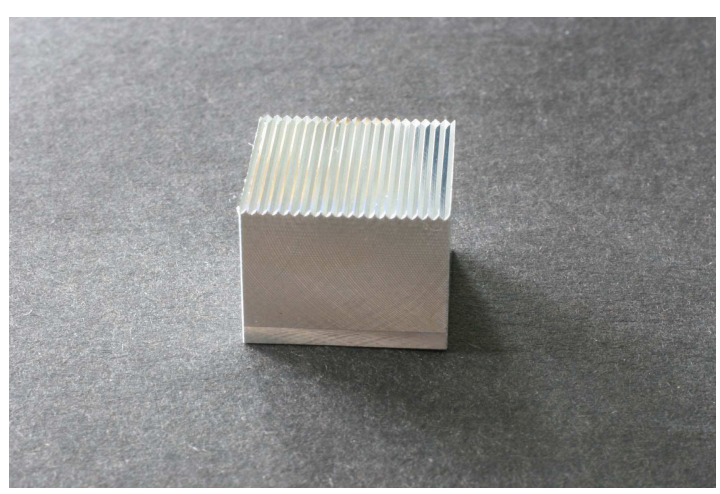

FIG. 5 Photograph of the retroreflector array used in the experiments.

The number of facets of this particular device is $K=19$, i.e. the aperture is approximately $12 \mathrm{~mm}$. For illumination, a white light source was used. Light from the source was transmitted through a pinhole and collimated. Due the finite width of the pinhole a small divergence of the collimated beam occurs which, however, did not affect the resolution of the spectrometric setup negatively since the bands could be well observed. We present experimental results in Figure 6 which show photographs and intensity plots of the first diffraction order for different tilt angles $\alpha=0,50^{\prime}, 60^{\prime}, 70^{\prime}, 80^{\prime}$.

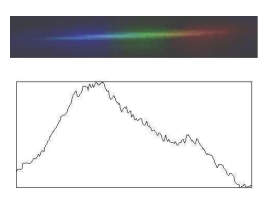

a)



d)

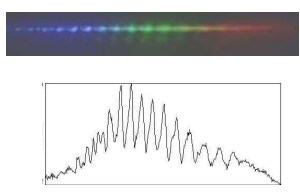

b) c)

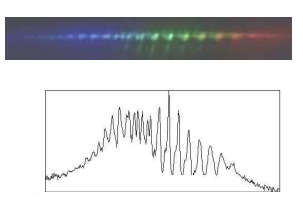

FIG. 6 Observation of modulated spectra for different tilt angles $\alpha$ of the RA. By increasing, the generated time delays are increased and modulation shifts gradually from the blue to the red part of the spectrum as can be seen from figures a) - e). The tilt angles were from a) to e) $0,50,60,70,80 \mathrm{~min}$, respectively. The axes on the intensity plots are intensity in arbitrary units vs. wavelength.

Ideally, for $\alpha=0$ no modulation should occur across the spectrum. As the angle is increased, one notice that a finer modulation across the spectrum occurs and shifts from smaller towards larger wavelengths. This is, of course, due to the fact 
that the generated time delays increase with the angle $\alpha$. In Figure 7 we demonstrate the influence of the filtering by using a mask with a linear variation of the amplitude across the aperture.

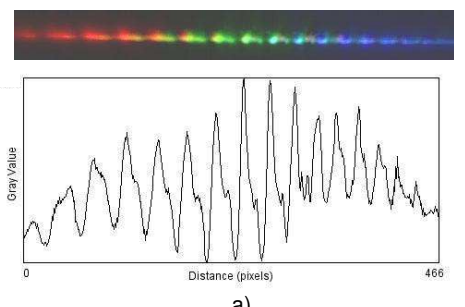

a)

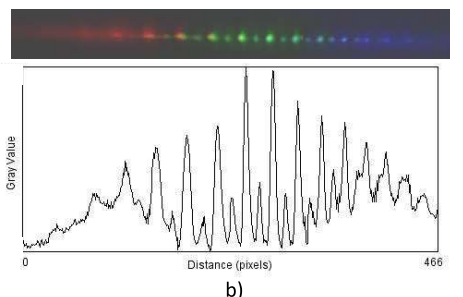

b)

FIG. 7 When an aperture is inserted between grating and the retroreflector, the in terference fringes are filtered. Thus in the frequency domain, some of the frequency components would be removed. The axes on the intensity plots are intensity in arbitrary units vs. wavelength.

In Figure 7a we see the spectrum for a given angle $\alpha$, without the mask, so that the maximum modulation takes place at the center of the spectrum. In Figure $7 \mathrm{~b}$ for the same angle we see the output with the mask present. As we derived in the previous section (Eq. (10)), an amplitude wedge-like mask acts as a differential filter with amplitude-shifted modulation. From Eq. (10), we expect to see sharper and partially shifted peaks in Figure $7 \mathrm{~b}$ as compared to Figure $7 \mathrm{a}$.

The experimental results shown in this section have a strong similarity with the so-called "Talbot bands". Talbot's bands were originally described for an experiment where the illumination beam of a grating or prism spectrometer is modified by inserting a glass plate halfway into the aperture [7]. Recently, an explanation of this experiment was given in terms of a refractive-diffractive dispersion effect and a theory was presented to calculate the intensity pattern of the spectrum [8]. In this article, in particular, the additional use of introducing also reflective time delays was suggested. We will now discuss the relationship of the RA setup described here and the classical Talbot bands experiment

\section{RELATIONSHIP WITH TALBOT'S B A N D S}

In this section, we consider not only the optical performance of the RA by itself, but rather the combination of RA and grating spectrometer. A qualitative argument for the occurrence of the band structure of the spectrum that we showed in the previous section uses a consideration propagation delays. Assume we send the short pulse through the setup, then a sequence of pulses will arrive in the output plane. The sequence is generated by the RA and also by the grating G. Bands occur only if the pulses arrive in the output plane approximately at the same time i.e., if the reflective time delay $\tau_{r}$ is matched by a diffractive delay $\tau_{d}$ introduced by the grating, i.e. if

$$
\tau_{\text {total }}=\tau_{r}+\tau_{d} \approx 0 .
$$

This argument is visualized by Figure 8 .

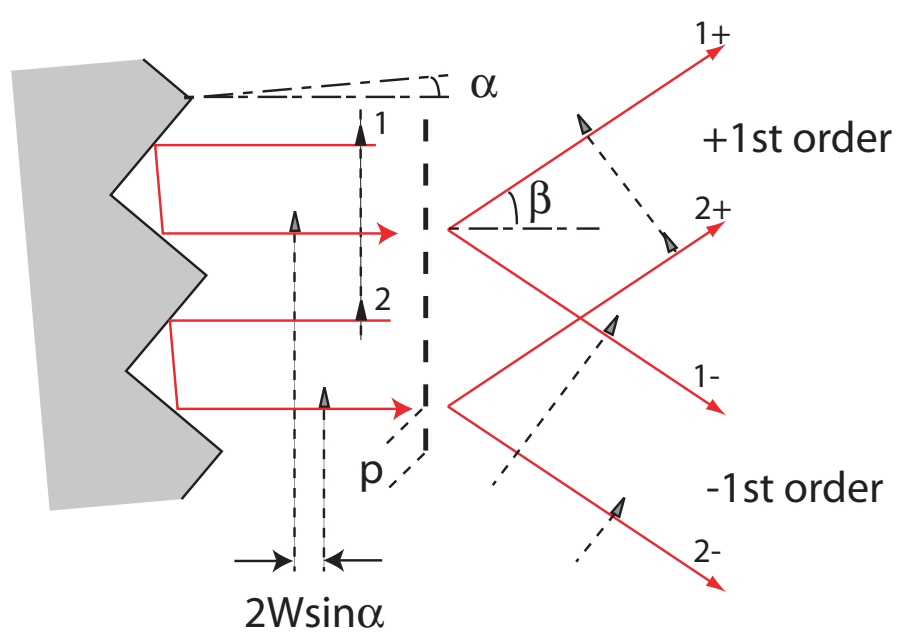

FIC. 8 Reflective and diffractive time delays occurring in the setup. The pulse numbered " 2 ", experiences a reflective time delay $\tau_{r}$ from the RA facet relative to pulse " 1 ". Each pulse is split up into two pulses by grating $G$. In the direction of the +1 st order, pulse " $1+$ " is delayed, so that under suitable conditions pulse " $2+$ " can catch up which leads to interference and a modulation of the 1st order. In the direction of the -1st order, however, pulse "2-" is further delayed with respect to pulse "1-" so that the pulse will not overlap, hence no interference fringes occur in the -1st order.

From the figure it is obvious that the condition expressed in Eq. (12) for the small angles, can only be satisfied for one of the first diffraction order, not simultaneously for both of them. Figure 8 shows that for the upward detour, the pulse denoted by number " 2 " is delayed two times first retardation due to reflection form the RA and second, detour on the way downward, hence two pulses can not interfere. But for upward case, the pulse of specified by " $1+$ " experiences the time delay due to the grating, therefore the pulse so called " $2+$ " can catch up and overlap with the pulse " $1+$ ". Now we calculate the intensity of the diffracted light traveling in direction $\beta$. We follow the analysis presented in Refs. [8,9]. The field of a light wave propagating in that direction is $s(t, \beta)$. The intensity observed in that direction is

$$
I(\beta)=\int|s(t, \beta)|^{2} \mathrm{~d} t=\int|S(\nu, \beta)|^{2} \mathrm{~d} \nu .
$$

The right identity follows from Parseval's theorem. From Eqs. (8) and (13), one can find

$$
I(\beta)=\int\left|S_{0}(v)\right|^{2}|H(v, \beta)|^{2} \mathrm{~d} v,
$$

where $S_{0}(v)$ and $H(v, \beta)$ indicate for the power spectrum of the incident beam and transfer function of the total discrete system RA + grating along direction of $\beta$, respectively. It can be written as follows [8]

$$
H(v, \beta)=\sum_{k=0}^{K-1} \sum_{m=0}^{M-1} \exp \left\{-2 \pi i v\left[(m+k M) \tau_{d}-k \tau_{r}\right]\right\} .
$$




$$
\begin{aligned}
& =\sum_{k=0}^{K-1} \exp (-2 \pi i k \varphi) \sum_{m=0}^{M-1} \exp \left(-2 \pi i m v \tau_{d}\right) \\
& =F(\nu, \beta) G(\nu, \beta)
\end{aligned}
$$

where $\varphi$ is defined as

$$
\varphi=v\left(M \tau_{d}-\tau_{r}\right),
$$

and $|G(\nu, \beta)|^{2}$ is

$$
\begin{aligned}
|G(\nu, \beta)|^{2} & =\left|\sum_{m=0}^{M-1} \exp \left(-2 \pi i m v \tau_{d}\right)\right|^{2} \\
& =\frac{\sin ^{2}\left(\pi M v \tau_{d}\right)}{\sin ^{2}\left(\pi v \tau_{d}\right)}
\end{aligned}
$$

which describes the well-known diffraction pattern generated by a grating, also

$$
|F(\nu, \beta)|^{2}=\frac{\sin ^{2}(\pi K \varphi)}{\sin ^{2}(\pi \varphi)},
$$

is the modulation of the diffraction pattern that it is known as Talbot's bands. From Eq. (18) also it is clear that in case $\sin \alpha=\sin \beta$ then the maximum modulation takes place i.e. $\alpha=\beta$. An amplitude wedge-like mask changes Eq. (10) into

$$
\begin{aligned}
F_{\text {lin }}(\nu, \beta) & =\sum_{k=1}^{K}\left(h_{0}+k \Delta h\right) \exp (-2 \pi i k \varphi) \\
& =h_{0} F(\nu, \beta)+\frac{i \Delta h}{2 \pi} \frac{\partial F(\nu, \beta)}{\partial \varphi} .
\end{aligned}
$$

\section{COMPARISON WITH CONVEN- TIONAL PULSE SHAPER}

As suggested in this article, the RA may be a useful device for temporal filtering or shaping of short optical pulses. Although it is not a mature approach it may be useful to compare with conventional techniques. The most common approach to optical pulse shaping uses a pair of diffraction gratings known as "zero dispersion pulse compressor [10,11]. It is shown in Figure 9.



FIG. 9 Sketch of the conventional setup for the processing of fs-pulses, compare, e.g., C. Froehly [10] and A. M. Weiner [11]. The input pulse is spectrally dispersed by grating $G_{1}$, the spectrum is accessible in the back focal plane of lens $L_{1}$. Lens $L_{2}$ and grating $\mathrm{G}_{2}$ generate an output signal.

It consists of a pair of diffraction gratings and lenses and a pulse shaping mask. The first diffraction grating disperses the frequency components of an incident ultra short beam. The frequency components are spatially separated along one dimension in the back focal plane of a lens. A desired amplitude or phase mask is inserted midway between the two lenses at a maximal spatial separation of optical spectral components. After the mask, the second lens and grating recombine the different frequencies into a single collimated beam. The output pulse is given by the Fourier transform of the pattern transferred by the mask onto the spectrum. As it is apparent from Figure 9 this configuration is ideal if the combination of the gratings and lenses is free of dispersion. For this, the lenses must be thin and free of aberration, dispersion in passing through the whole elements should be small, and the gratings should have a flat spectral response. In practice there are several approximations and limitations on these assumptions. The central feature of the conventional pulse shaper is that the different frequency components can be accessed individually. A modulator can be used to perform certain filtering operations. Liquid crystal devices or static masks may be used consisting of several hundred elements.

In comparison, in our device the filtering operation is implemented in the time domain by introducing the delays among the facets. As explained earlier, a mask is used to realize a specific filter function. The mask may also be static or dynamic. The number of facets used determines the order of the filter function. If $\mathrm{N}$ facets are used then a linear filter of $\mathrm{N}$-th order is implemented. It is noteworthy that this number does not correspond directly with the number of modulator elements in the conventional pulse shaper. A practical advantage of our method may lie in the fact that it is not necessary to split up the frequency components to access them in the spatial domain. Rather different time delays can be generated by a suitable pitch of the RA. Furthermore, since the RA already implements the filter one may save other optical elements. Although, we have shown the basic configuration of our setup using classical beam splitter and with transmissive lenses, they may be also realized in reflection in order to minimize unwanted dispersion.

\section{C O N CLUSION AND OUTLOOK}

We have described and demonstrated the use of a retroreflector array as a temporal filter. Its performance was described theoretically by using the concept of the transfer function. Furthermore, the relationship to the classical Talbot bands was pointed out. Experimental results were shown that demonstrate the predicted performance on the optical spectrum. General filtering operations can be achieved by adding a mask (phase and/or amplitude).

For a practical implementation, an integrated version rather than a bulk optical setup may be of interest. For systems integration, optical and mechanical functions need to be combined. The optics may be implemented using the concept of planar integrated free-space optics [12]. Currently, research is ongoing to demonstrate such an integrated setup.

Remark: This work was carried at the Fernuniversität Hagen while A. Sabatyan was on leave from the IASBS. 


\section{References}

[1] R. Holzwarth, Th. Odem, T. W. Hänsch, J. C. Knight, W. J. Wadsworth, P. St. J. Russell, "Optical frequency synthesizer for precision spectroscopy" Phys. Rev. Lett. 85, 2264-2267 (2000).

[2] B. E. Nelson, A. Keeler, D. Agarwal, N. C. Helman, D. A. B. Miller, "Wavelength division multiplexed optical interconnect using short pulses" IEEE. J. Select. Topics Quantum Electron. 9, 486-491 (2003).

[3] T. Kurokawa, K. Okamoto, K. Naganuma, H. Takenouchi, Y. Inoue and M. Ishii, "Time-space-conversion optical signal processing using arrayed-waveguide grating," Electron. Lett. 33, 1890-1891 (1997).

[4] B. E. Little, S. T. Chu, H. A. Haus, J. Foresi, and J. P Laine, "Microring resonator channel dropping filters" J. Lightwave Technol. 15, 998-1005 (1997).

[5] J. Jahns, A. W. Lohmann, "Temporal filtering by double diffraction" Appl. Opt, 43, 4339-4344 (2004).
[6] R. G. Lyons, Understanding digital signal processing, (Prentice. Hall. PTR 2001).

[7] H. F. Talbot, "An experiment on the interference of light" Phil. Mag. 10, 364 (1837).

[8] J. Jahns, A. W. Lohmann, M. Bohling, "Talbot bands and temporal processing of optical signals" J. Eur. Opt. Soc-RP. 1, 06001 (2006).

[9] G. Mínguez-Vega, J. Jahns, "Temporal processing with the montgomery interferometer" Opt. Commun. 236, 45-52 (2004).

[10] C. Froehly, B. Colombeau, M. Vampouille, "Shaping and analysis of picosecond pulsesin", in Progress in optics, E. Wolf, ed., 20, 63-153 (North-Holland, Amsterdam, 1983).

[11] A. M. Weiner, J. P. Heritage, E. M. Kirschner, "Encoding and decoding of femtosecond pulses," Opt. Lett. 13 300-302 (1988).

[12] J. Jahns, A. Huang, "Planar integration of free-space optical components" Appl. Opt. 28, 1602-1605 (1989). 